\title{
Comportamiento productivo e incidencia de diarrea en cerdos posdestete suplementados con polvo mixto de hojas de plantas con propiedades nutracéuticas
}

\section{Growth performance and diarrhea incidence in post- weaning pigs, supplemented with plants mixed leaves powder, with nutraceutical properties}

Fecha de recepción: 8 de enero de 2017 Fecha de aprobación: 2 de junio de 2017

\author{
Roisbel Aroche-Ginarte ${ }^{1}$ \\ Yordan Martínez-Aguilar ${ }^{2}$ \\ Lazara Ayala-González ${ }^{3}$ \\ Román Rodríguez-Bertot ${ }^{4}$ \\ Yuri Rodríguez-Fraga ${ }^{5}$
}

DOI: http://doi.org/10.19053/01228420.v14.n2.2017.7145

\section{Resumen}

Para evaluar el efecto de la suplementación dietética con polvo mixto de hojas de plantas con propiedades nutracéuticas (40\% A. occidentale, $20 \%$ M. oleifera, $20 \%$ M. citrifolia y $20 \%$ P. guajava) en el comportamiento productivo y en la incidencia de diarrea en cerdos posdestete, se utilizaron 150 cerdos (Yorkland x Yorkshire) de 33 días de edad, con peso vivo promedio de $7.74 \mathrm{~kg} \pm 0.71$. Se aplicó un diseño completamente aleatorizado con seis repeticiones por tratamiento y cinco cerdos por repetición, durante 42 días. Los tratamientos fueron, T0: Dieta Basal (DB) sin Antibiótico Promotor de Crecimiento (APC); T1: DB + APC (Labionor $5 \mathrm{~mL} / \mathrm{kg}$ de pienso); T2: $\mathrm{DB}+0.5 \%$ de polvo mixto; T3: $\mathrm{DB}+1.0 \%$ polvo mixto, y T4: $\mathrm{DB}+1.5 \%$ de polvo mixto. La viabilidad mejoró $(\mathrm{P}<0.05)$ con la suplementación dietética del polvo mixto y el APC, comparada con el T0; los T3 y T4 incrementaron $(\mathrm{P}<0.05)$ el peso vivo final con relación al T0 y al T1, y el T4 mostró $(\mathrm{P}<0.05)$ la mejor ganancia media diaria y conversión alimenticia, con diferencias significativas $(\mathrm{P}<0.05)$ con el T0 y el T1. Las suplementaciones del polvo mixto y del APC disminuyeron $(\mathrm{P}<0.05)$ la incidencia de la diarrea en los cerdos posdestete. Se recomienda la

\footnotetext{
M. Sc. Universidad de Granma (Bayamo, Cuba). rarocheg@udg.co.cu. Ph. D. Universidad de Granma (Bayamo, Cuba). yma@ica.co.cu.

Ph. D. Instituto de Ciencia Animal (San José de las Lajas, Cuba). layala@ica.co.cu. ORCID: 0000-0002-0739-3463.

Ph. D. Universidad de Granma (Bayamo, Cuba).rrodriguezb@udg.co.cu.

Instituto de Ciencia Animal (San José de las Lajas, Cuba). yuri@ica.co.cu.
} 
suplementación dietética de $1.0 \%$ del polvo mixto de hojas de plantas con propiedades nutracéuticas como alternativa a los APC, para incrementar los indicadores productivos y disminuir la incidencia de diarrea en los cerdos posdestete.

Palabras clave: cerdos; nutracéutico; porcicultura; productividad porcina; suplementación alimentaria.

\begin{abstract}
To evaluate the plants mixed leaves powder dietary supplementation with nutraceutical properties effect by (40\% A. occidentale, $20 \%$ M. oleifera, $20 \%$ M. citrifolia y $20 \%$ P. guajava) on growth performance and diarrhea incidence in post-weaning pigs, were used 150 piglets, with an average body weight of 7.74 $\mathrm{kg} \pm 0.71$ (Yorkland $x$ Yorkshire) of 33 days old. A completely randomized design with six replicates per treatment, and five piglets per replicate during 42 days was applied. Treatments were T0: A basal diet (BD) without antibiotic growth promoter (AGP); (T1): BD + AGP (Labionor $5 \mathrm{~mL} / \mathrm{kg}$ of fodder); (T2): BD + 0.5 $\%$ of mixed powder, (T3): BD+1.0 \% of mixed powder, and (T4): BD+1.5\% of mixed powder.
\end{abstract}

Viability improved $(\mathrm{P}<0.05)$ with dietary supplementation of the mixed leaves powder and AGP compared with the T0. Also, the T3 and T4 increased $(\mathrm{P}<0.05)$ the final alive body weight,related to T0 and T1, as well as, the $\mathrm{T} 4$ which showed the best $(\mathrm{P}<0.05)$ average daily gain and feed conversion ratio, with significant differences $(\mathrm{P}<0.05)$ with the T0 and T1.

The mixed powder supplementation and AGP decreased $(\mathrm{P}<0.05)$ the diarrhea incidence in post-weaning pigs. It is recommended the dietary supplementation of $1.0 \%$ mixed leaves powders of plants with nutraceutical properties as alternative to the AGP, in order to increase the growth performance indexes and diminish the diarrhea incidence in post-weaning pigs.

Keywords: food supplementation; mixed powder; nutraceutical; pig production.

\title{
Como citar:
}

Aroche-Ginarte R., Martínez-Aguilar Y., Ayala-González L., Rodríguez-Bertot R., Rodríguez-Fraga Y. Comportamiento productivo e incidencia de diarrea en cerdos posdestete suplementados con polvo mixto de hojas de plantas con propiedades nutracéuticas. Rev. Cien. Agri. 2017; 14(2): 19-26. 


\section{Introducción}

Tras el destete, la alimentación de los cerdos cambia bruscamente: de una alimentación basada en leche materna pasa a alimento sólido, lo que, acompañado de otros factores como la separación de la madre y el enfrentamiento con cerdos de otras camadas, desencadena el síndrome de estrés en esta etapa (1). Así, estos trastornos en la homeostasis reducen el consumo diario de nutrientes y energía (2), y provocan un desequilibrio en la microflora intestinal, lo que aumenta las oportunidades de los patógenos para colonizar el intestino $(3,4)$.

Por lo general, en la primera semana después del destete se observa una depresión del crecimiento, lo que repercute negativamente en la etapa de preceba y trae consigo grandes pérdidas (5). Para corregir estos problemas en la producción intensiva de cerdos, desde hace más de 50 años se han utilizado los antibióticos subterapéuticos, o antibióticos promotores de crecimiento (APC), en el pienso (6) suministrado.

Actualmente, muchos países prohíben el uso de APC; sin embargo, otros lo utilizan con gran amplitud. El uso indiscriminado de los APC ha provocado resistencia microbiana y resistencias cruzadas en otros microrganismos; la premisa presente y futura de las investigaciones es buscar fuentes naturales como alternativa a los APC en la producción de cerdos, sobre todo para las etapas más críticas, como el destete. Dentro de estas fuentes están algunas plantas con propiedades medicinales, como:

Anacardium occidentale (marañón): es un árbol tropical de crecimiento rápido, nativo de Brasil. Estudios en esta planta han referido propiedades antiinflamatorias, antibacterianas, analgésicas, anticoagulantes, antiespasmódicas y astringentes (7).

Psidium guajava (guayaba): es un árbol nativo de América Tropical; sus hojas y corteza son utilizadas como fitopreparados en animales $y$ humanos por sus propiedades antibacterianas, antieméticas, antiinflamatorias, antihelmínticas, antisépticas, antitóxicas, astringentes, carminativas, antiespasmódicas y tónicas (8).

Moringa oleifera: es la especie más conocida del género Moringa; es un árbol originario del sur del Himalaya, el nordeste de la India, Bangladesh, Afganistán y Pakistán; se encuentra diseminado en una gran parte del planeta, y en América Central fue introducida en los años 1920 como planta ornamental y para cercas vivas (9); Price (10) la recomienda para contrarrestar la desnutrición de los niños y como alimento humano en general.

Morinda citrifolia (Noni): pertenece a la familia Rubiaceae; es un árbol oriundo de la zona tropical de Asia del Sur, Australia, Hawaii y las islas de la Polinesia francesa; sus frutas, hojas, semillas, corteza y raíces han sido usadas tradicionalmente para prevenir o mejorar varias enfermedades, como la artritis, infecciones, resfriados, cáncer y diabetes $(11,12)$.

El suministro de estas plantas en la dieta de los cerdos destetados mejora los indicadores productivos, la digestibilidad de nutrientes y la respuesta inmune, y disminuye el síndrome diarreico; además, su adición se fundamenta en la seguridad de su inclusión y su nula residualidad $(13,14)$.

El objetivo del presente trabajo fue evaluar el efecto de la suplementación dietética del polvo mixto de hojas de plantas con propiedades nutracéuticas (40\% A. occidentale, $20 \% \mathrm{M}$. oleifera, $20 \% \mathrm{M}$. citrifolia y $20 \%$ P. guajava) en el comportamiento productivo e incidencia de diarrea en cerdos posdestete.

\section{Materiales y métodos}

Se tomaron hojas de 20 árboles de A. occidentale, $P$. guajava y $M$. citrifolia con más de cinco años de establecimiento en la zona de Peralejo, Bayamo, Granma, Cuba, caracterizada por una topografía Ilana y suelo ferralítico. Además, se recolectaron hojas de 10 árboles de M. oleifera en áreas experimentales del Instituto de Ciencia Animal (ICA), caracterizadas por suelo ferralítico rojo típico, de rápida desecación y perfil uniforme (15). 
Las hojas se identificaron en el Departamento de Botánica de la Facultad de Ciencias Agrícolas de la Universidad de Granma; además, para la recolección se tuvo en cuenta la diversidad del tamaño y estructura de las hojas (9). Las muestras de $A$. occidentale, $P$. guajava y $M$. oleifera se deshidrataron a la sombra durante tres días consecutivos, y las de M. citrifolia, durante siete días, y se removieron dos veces por día; luego, se depositaron en una estufa (WSU 400, alemana) con recirculación de aire durante una hora a 60 ${ }^{\circ} \mathrm{C}$; posteriormente, se trituraron en un molino de martillo de cuchillas paralelas, a $1 \mathrm{~mm}$ de granulometría. Las muestras se almacenaron a temperatura ambiente en bolsas de plástico totalmente herméticas (16).

El experimento se realizó en la unidad experimental porcina del ICA, durante 42 días, con 150 cerdos (Yorkland x Yorkshire) destetados a los 33 días, según diseño completamente aleatorizado, con cinco tratamientos, seis repeticiones y cinco cerdos por corral. Los tratamientos consistieron de una dieta basal (DB) sin APC (T0); DB con APC -Labionor $5 \mathrm{~mL} / \mathrm{kg}$ de pienso- (T1); DB + $0.5 \%$ de polvo mixto $-40 \% \mathrm{~A}$. occidentale, $20 \% \mathrm{M}$. oleifera, $20 \%$ M. citrifolia y $20 \%$ P. guajava(T2); $\mathrm{DB}+1.0 \%$ de polvo mixto (T3); $\mathrm{DB}+1.5$ $\%$ de polvo mixto (T4). El antibiótico utilizado fue suministrado por la empresa farmacéutica Labiofam (Cuba).

El alimento y el agua se ofertaron ad libitum en comederos tipo tolva y bebederos tipo tetina. La dieta se confeccionó a base de maíz y soya (Tabla I) y se formuló teniendo en cuenta los requerimientos según la norma de la NRC (17) y lo indicado en el Manual de Procedimientos Técnicos de la Crianza Porcina (18).
Tabla I. Composición de la dieta (BH).

\begin{tabular}{|l|l|}
\hline \multicolumn{1}{|c|}{ Materia prima } & \% \\
\hline Harina de maíz & 62.06 \\
\hline Harina de soya & 35.00 \\
\hline Carbonato de calcio & 1.00 \\
\hline Fosfato dicálcico & 1.00 \\
\hline Sal común & 0.25 \\
\hline Premezcla de minerales y vitaminas* & 0.50 \\
\hline Cloruro de colina & 0.19 \\
\hline Aporte calculado & \\
\hline PB $(\%)$ & 20.00 \\
\hline ED $(\mathrm{kcal} / \mathrm{kg})$ & 3270 \\
\hline Ca $(\%)$ & 0.60 \\
\hline P $(\%)$ & 0.30 \\
\hline
\end{tabular}

* Fuente: NRC (1998).

Los cerdos se pesaron, individualmente, al destete (33 días) y al final del experimento (75 días), con una pesa de brazo o machete con precisión \pm 1 g. La viabilidad se computarizó por la cantidad de cerdos durante la etapa experimental entre los que se alojaron al inicio del experimento. Además, se calculó la conversión alimenticia como la cantidad de alimento ingerido para ganar $1 \mathrm{~kg}$ de peso vivo. Para determinar la incidencia de diarrea (ID) se observó si hubo presencia de esta durante los 42 días experimentales, y se utilizó la fórmula de Hampson (19):

$$
\text { ID }=\frac{\text { Número de diarreas }}{\text { cantidas de animales } x \text { total de días }} \times 100
$$


Los datos se procesaron mediante análisis de varianza (Anova) de clasificación simple; antes de realizar el análisis de varianza se procedió a verificar la normalidad de los datos, por la prueba de Kolmogorov Smirnov, y para la uniformidad de la varianza, la prueba de Bartlett. En los casos necesarios se empleó la prueba de Duncan para determinar las diferencias entre medias, según el software estadístico SPSS versión 18.0. La viabilidad se analizó por comparación de proporciones en el software estadístico COMPARPRO 1.0.

\section{Resultados y discusión}

En la Tabla II se muestra el efecto de la suplementación dietética con polvo mixto de plantas medicinales en los indicadores productivos de cerdos en crecimiento. Los resultados de la viabilidad demuestran que esta etapa de la vida de los cerdos es muy compleja, ya que solo un tratamiento (T2) mostró $100 \%$ de viabilidad, aunque no se encontraron diferencias significativas $(\mathrm{P}<0.05)$ entre las adiciones del polvo mixto y el antibiótico. Estos resultados demuestran la inocuidad del producto utilizado durante 42 días.

Se ha demostrado que las plantas utilizadas en este estudio tienen propiedades nutracéuticas, tales como antibacterianas, antioxidantes y antiinflamatorias (20), por lo que su adición en pequeñas concentraciones en las dietas influye positivamente en la viabilidad. Asimismo, investigaciones de Ayala et al. (21) con fitobióticos mostraron los beneficios de los productos naturales, por sus propiedades reguladoras de las funciones orgánicas, importante para maximizar la expresión genética de los animales.

Tabla II. Efecto de la suplementación dietética con polvo mixto de plantas medicinales en indicadores productivos de cerdos en crecimiento.

\begin{tabular}{|c|c|c|c|c|c|c|c|}
\hline \multirow[t]{2}{*}{ Ítems } & \multicolumn{5}{|c|}{$\begin{array}{c}\text { Suplementación dietética con polvo mixto de plantas } \\
\text { medicinales }(\%)\end{array}$} & \multirow[t]{2}{*}{$\mathrm{EE} \pm$} & \multirow[t]{2}{*}{ Valor de $\mathbf{P}$} \\
\hline & T0 & T1 & T2 & T3 & T4 & & \\
\hline Viabilidad (\%) & $83.3^{\mathrm{b}}$ & $96.7^{\mathrm{a}}$ & $100^{\mathrm{a}}$ & $96.7^{\mathrm{a}}$ & $96.7^{\mathrm{a}}$ & 4.89 & 0.041 \\
\hline PI (kg) & 7.52 & 7.39 & 7.74 & 8.17 & 7.86 & 0.26 & 0.380 \\
\hline PF (kg) & $\begin{array}{l}20.24^{\mathrm{c}} \\
(0.68)\end{array}$ & $\begin{array}{l}21.30^{\mathrm{bc}} \\
(0.63)\end{array}$ & $\begin{array}{l}22.92^{a b} \\
(0.62)\end{array}$ & $\begin{array}{l}23.56^{\mathrm{a}} \\
(0.63)\end{array}$ & $\begin{array}{l}23.56^{\mathrm{a}} \\
(0.63)\end{array}$ & & 0.001 \\
\hline GMD (g/cerdo/día) & $297^{c}$ & $330^{\mathrm{bc}}$ & $361^{\mathrm{ab}}$ & $366^{\mathrm{ab}}$ & $375^{\mathrm{a}}$ & 12.23 & 0.001 \\
\hline CA (kg/kg) & $3.14^{c}$ & $2.73^{b}$ & $2.48^{\mathrm{ab}}$ & $2.44^{\mathrm{ab}}$ & $2.39^{a}$ & 0.11 & 0.002 \\
\hline
\end{tabular}

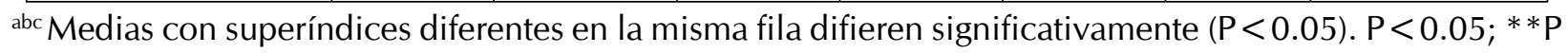
$<0.01$; ** $\mathrm{P}<0.001$.

PI: Peso inicial; PF: Peso final; GMD: Ganancia media diaria; CA: conversión alimenticia

Se observa, además, que los T3 y T4 mostraron los mayores pesos vivos $(\mathrm{P}<0.05)$ al finalizar el experimento, en comparación con los T0 y T1; esto pudo deberse a que el uso de mezclas de plantas con propiedades nutracéuticas favorece los procesos digestivos, inmunes y antiinflamatorios, sobre todo, por la sinergia de sus compuestos químicos, efecto ya demostrado por Kong et al. (20) con polvos mixtos de plantas medicinales asiáticas.

Asimismo, las diferencias en el peso final podrían estar asociadas a un mejor ambiente microbiano a nivel intestinal; en este sentido, Ding et al. (22), utilizando un polvo mixto de plantas medicinales en las dietas de los cerdos, encontraron un incremento de la respuesta productiva por una mayor colonización de bacterias acido lácticas y exclusión competitiva.

Por otro lado, la presencia de $A$. occidentale y $P$. guajava en el polvo mixto pudo tener influencia en estos resultados, ya que son portadores de sustancias polifenólicas, como los taninos. Según Okuda (23), algunos grupos de taninos actúan en el metabolismo del ácido araquidónico en los leucocitos, lo que 
influye de manera importante en la reversión de procesos inflamatorios. Lo anterior permite prever una respuesta más rápida del sistema inmune ante el ataque de bacterias patógenas como $E$. coli, además de una reducción de la inflamación de la mucosa intestinal.

Asimismo, los alcaloides detectados en la M. citrifolia y $M$. oleifera (24) tienen efectos benéficos, en pequeñas proporciones, y son potentes bactericidas contra cepas de Staphylococcus aureus y E. coli, lo que pudo influir en el peso vivo de los cerdos en el T3 y T4, por una mayor salud intestinal, con influencia positiva en la digestibilidad de los nutrientes (principalmente aminoácidos esenciales y minerales) (25).

Otro indicador que mejoró $(\mathrm{P}<0.05)$ con la suplementación dietética del polvo mixto fue la GMD, sobre todo con los T3 y T4, cuyos valores coincidieron con los del peso vivo (Tabla 2). Estos resultados concuerdan con los presentados por Kong et al. (26) y Ding et al. (22), que encontraron un incremento de la GMD en cerdos jóvenes debido al efecto de los compuestos fitoquímicos de las plantas utilizadas. Según Kamel (27), Balunas y Kinghorn (28) y Athanasiadou et al. (29), los extractos o compuestos bioactivos de plantas y hierbas usados en la alimentación animal se definen como componentes de origen vegetal que al ser incluidos mejoran la productividad a través de un mayor aprovechamiento de los nutrientes y de la eliminación de patógenos residentes en el intestino.

A pesar de que todos los tratamientos consumieron el alimento suministrado, la conversión alimenticia mejoró cuando se usaron los tres niveles de polvo mixto en las dietas de los cerdos, principalmente el T4, en comparación con el T0 y el T1, expresada en mayor peso vivo; esto se asocia, básicamente, a que la dieta basal se formuló isoenergética e isoproteica, lo que no repercutió en el consumo de alimento. Este indicador importante en la producción porcina demuestra la efectividad de las plantas medicinales como alternativa a los antibióticos dietéticos.

Por otro lado, los cerdos pueden asimilar mayor cantidad de metabolitos secundarios que las aves, especie en la cual se han investigado las plantas utilizadas en este estudio, por lo que sería recomendable realizar estudios con mayores niveles de suplementación del polvo mixto de las plantas empleadas. Sin embargo, un exceso de sustancias bioactivas en las dietas de los cerdos puede provocar síntomas relacionados a factores antinutricionales, lo que influye en una disminución del peso vivo (30).

Un importante descubrimiento de este estudio es que la suplementación dietética con polvo mixto de plantas con propiedades medicinales redujo la incidencia de la diarrea de cerdos en crecimiento (Figura 1), en 42 días de experimento, al punto de que se puede afirmar que el efecto antidiarreico de este nuevo producto natural fue similar al del APC.

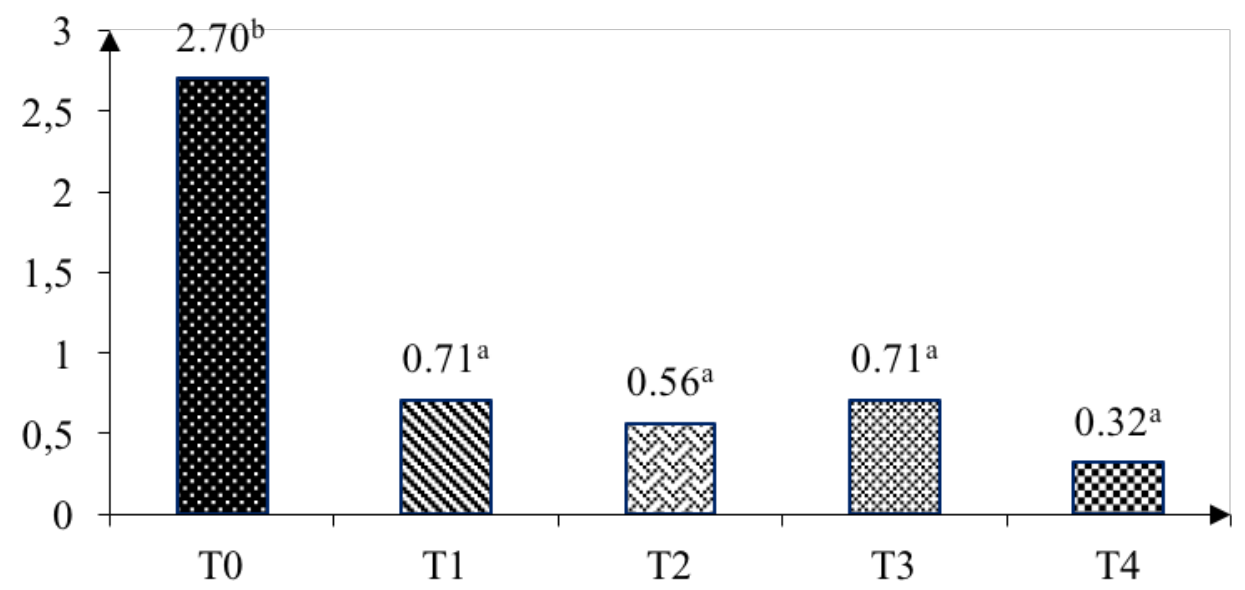

Fig. 1. Efecto de la suplementación dietética con polvo mixto de plantas medicinales en la incidencia de la diarrea de cerdos en crecimiento. 
El síndrome diarreico es provocado por la pérdida de agua y electrólitos en heces semilíquidas y líquidas; ocurre debido, principalmente, a la proliferación de $E$. coli y otras bacterias patógenas intestinales en cerdos destetados (7 a 14 días posdeteste) $(20,26)$. Autores como Martínez et al. (8) y Pérez et al. (31) han reportado una disminución de la incidencia de diarrea en cerdos, aves, conejos y humanos, al utilizar terapéuticamente polvos medicinales de $A$. occidentale y $P$. guajava, principalmente, relacionado al efecto antidiarreico, antinflamatorio y bactericida de sus metabolitos secundarios (en especial taninos y coumarinas), por lo que su mezcla en las dietas de los cerdos podría tener un efecto aún mayor, por la sinergia de estos metabolitos secundarios, regulando las funciones orgánicas, el $\mathrm{pH}$ y el peristaltismo intestinal. Resultados similares han sido encontrados por Kong et al. (20) y Ding et al. (22) en cerdos en crecimiento.

Como se refirió anteriormente, el posible efecto antimicrobiano de las diferentes concentraciones empleadas de polvo mixto podría actuar como reguladores de la microbiota intestinal (principalmente E. coli), sobre todo en esta etapa de vida, caracterizada por una alta proliferación de bacterias patógenas intestinales, lo que provoca mayor incidencia de diarrea (22). Estos resultados podrían deberse a la acción bacteriostática, bactericida y antioxidante de los taninos y otros compuestos polifenólicos presentes en las plantas utilizadas (26).

\section{Conclusiones}

Se recomienda la suplementación dietética de $1.0 \%$ del polvo mixto de hojas de plantas con propiedades nutracéuticas $(40 \%$ Anacardium occidentale, $20 \%$ Psidium guajava, $20 \%$ Morinda citrifolia y $20 \%$ Moringa oleifera) como alternativa a los APC, para incrementar los indicadores productivos y disminuir la incidencia de diarrea en los cerdos posdestete.

\section{Agradecimientos}

A los trabajadores de la unidad integral porcina del Instituto de Ciencia Animal, por su apoyo incondicional durante el desarrollo de la investigación, y al técnico en adiestramiento Denis Almanza Saavedra, por su colaboración.

\section{Referencias}

(1) Canibe N. Sistemas de alimentación y aditivos en piensos de iniciación. En: XXIII Curso de Especialización FEDNA, Madrid. 2007. Disponible en: http://www.acorex.es/es/pienso/Alimentaciondelechones.pdf.

(2) Pluske JR., Jae-Cheol K., Hansen CF., Mullan BP., Payne HG., Hampson $\mathrm{DJ}$., et al. Piglet growth before and after weaning in relation to a qualitative estimate of solid (creep) feed intake during lactation: A pilot study. Arch Anim Nutr. 2007; 61(6): 469-80. DOI: http://doi. org/10.1080/17450390701664249.

(3) Fuller R. Probiotics in man and animals. J App/ Bacteriol. 1989; 66(5): 365-78. DOI: http://doi.org/10.1111/j.1365-2672.1989.tb05105.x.

(4) Jensen BB. The impact of feed additives on the microbial ecology of the gut in young pigs. J Anim Feed Sci. 1998; 7: 45-64. DOI: http:// doi.org/10.22358/jafs/69955/1998.

(5) King RH-, Pluske JR. Nutritional management of the pig in preparation of weaning. En: Weaning the Pig-Concepts and Consequences. Holanda; 2003. pp. 37-51.

(6) Martin W., Verstegen A., Williams BA. Alternatives to the use of antibiotics as growth promoters in monogastric animals. Anim Biotechnol. 2002; 13(1): 113-127. DOI: http://doi.org/10.1081/ABIO120005774.

(7) Martínez Y., Martínez O., Liu G., Ren W., Rodríguez R., Fonseca Y., et al. Effect of dietary supplementation with Anacardium occidentale on growth performance and immune and visceral organ weights in replacement laying pullets. J Food Agric Environ. 2013; 13: 13521357.

(8) Bontempo P., Doto A., Miceli M., Mita L., Benedetti R. Psidium guajava L. anti-neoplastic effects: induction of apoptosis and cell differentiation. Cell Prolif. 2012; 45(1): 22-31. DOl: http://doi.org/10.1111/j.1365-2184.2011.00797.x.

(9) Foidl N., Mayorga L., Vázquez W. Utilización del marango (Moringa oleifera) como forraje fresco para ganado. FAO Anim Prod Health Pap. 1999; 341-50.

(10) Price ML. The Moringa tree. Educational Concerns for Hunger Organization (ECHO). 1985. Disponible en: http://www.echotech.org/ technical/technotes/moringabiomasa.pdf.

(11) Mc Clatchey W. From Polynesian healers to health food stores: Changing perspectives of Morinda citrifolia (Rubiaceae). Integr Cancer Ther. 2002; 1(2): 110-20. DOI: http://doi.org/10.1177/15347354 02001002002.

(12) Wang MY., West BJ., Jensen CJ., Nowicki D., Su C., Palu AK. Morinda citrifolia (Noni): a literature review and recent advances in Noni research. Acta Pharmacol Sin. 2002; 23(1): 1127-1141. 
(13) Cromwell GL. Why and how antibiotics are used in swine production. Anim Biotechnol. 2002; 13(1): 7-27. DOI: http://doi. org/10.1081/ABIO-120005767.

(14) Martínez Y., Martínez O., Olmos E., Siza S., Betancur C. Nutraceutical effect of Anacardium occidentale (AO) in diets of replacement laying pullets. JMVZ Córdoba. 2012; 17(3): 3125-3132.

(15) Rodríguez R. Estudio in vitro del valor nutritivo y de los efectos antinutricionales de cuatro leguminosas arbóreas tropicales con potencialidades como suplementos del Pennisetum purpureum. Tesis de Doctorado. España: Universidad de Zaragoza; 2010.

(16) Yin YL., Zhong HY., Huang RL., Chen CM., Li TJ., Pai YF. Nutritive value of feedstuffs and diets for pigs: I. Chemical composition, apparent ileal and fecal digestibility. Anim Feed Sci Technol. 1993; 44(1): 1-27. DOI: http://doi.org/10.1016/0377-8401(93)90034-H.

(17) NRC. Nutritional Requirements for Swine. National Academy Press. Washington, DC: National Research Council, Nutrient Requirements of Swine; 1998.

(18) IIP. Manual de procedimientos técnicos para la crianza porcina. La Habana, Cuba; 2015. 124 p.

(19) Hampson DJ. Alterations in piglet small intestinal structure at weaning. Res Vet Sci. 1986; 40(1): 32-40.

(20) Kong XF., Wu GY., Liao YP., Hou ZP., Liu HJ., Yin FG., et al. Effects of Chinese herbal ultra-fine powder as a dietary additive on growth performance, serum metabolites and intestinal health in early-weaned piglets. Livest Sci. 2007; 108(1): 272-275. DOI: http://doi.org/10.1016/j.livsci.2007.01.079

(21) Ayala L., Silvana N., Zocarrato I., Gómez S. Use of vulgar oregano (Origanum vulgare) as phytobiotic in fatting rabbits. Cuban / Agric Sci. 2011; 45(2): 159-161.

(22) Ding YY., Zhang CH., He XL., Huang L., Yin ZJ.. Growth performance responses and indicators of gastrointestinal health in early weaned pigs fed chinese herbal medicine additives-supplemented diets. J Anim Vet Adv. 2011; 10(12): 1580-1587. DOI: http://doi. org/10.3923/javaa.2011.1580.1587.

(23) Okuda T. Systematics and health effects of chemically distinct tannins in medicinal plants. Phytochemistry. 2005; 66(17): 2012-2031. DOI: http://doi.org/10.1016/j.phytochem.2005.04.023.

(24) Castillo A. Evaluación de la actividad antimicrobiana de los extractos de Mirabilis jalapa L. y Morinda citrifolia L. Tesis de Maestría. Cuba: Universidad de Granma; 2011.

(25) Ghasemi R., Zarei M., Torki M. Adding medicinal herbs including garlic (Allium sativum) and thyme (Thymus vulgaris) to diet of laying hens and evaluating productive performance and egg quality characteristics. Am J Anim Vet Sci. 2010; 5(2): 151-154. DOI: http://doi. org/10.3844/aja-vsp.2010.151.154.

(26) Kong XF., Wu GY., Yin YL., Liu HJ., Yin FG., Li TJ., et al. Dietary supplementation with Chinese herbal ultra-fine 3 powder enhances cellular and humoral immunity in early weaned piglets. Livest Sci. 2007; 108 (1): 94-98. DOI: http://doi.org/10.1016/j.livsci.2007.01.002.

(27) Kamel C. Natural plant extracts: classical remedies bring modern animal production solutions. Feed manufacturing in the Mediterranean region. Improving safety: from feed to food. En: Brufau J. Zaragoza: CIHEAM-IAMZ; 2001. p. 31-38.
(28) Balunas MJ., Kinghorn AD. Drug discovery from medicinal plants. Life Sci. 2005; 78(5): 431-441. DOI: http://doi.org/10.1016/j. Ifs.2005.09.012.

(29) Athanasiadou S., Githiori J., Kyriazakis I. Medicinal plants for helminthes parasite control: facts and fiction. Animal. 2007; 1(9): 13921400. DOI: http://doi.org/10.1017/S1751731107000730.

(30) Savón L., Scull I., Martínez M. Integral foliage meal for poultry feeding. Chemical composition, physical properties and phytochemical screening. Cuban J Agric Sci. 2007; 41: 359-369.

(31) Pérez RM., Mitchell S., Vargas R. Psidium guajava: A review of its traditional uses, phytochemistry and pharmacology. J Ethnopharmacol. 2008; 117(1): 1-27. DOI: http://doi.org/10.1016/j.jep.2008.01.025. 